\title{
SELF E CONTEÚdO N ÃO CONCEITUAL DA PERCEPÇÃO: A PERSPECTIVA ANTI-REPRESENTACIONISTA ACERCA DA EXPERIÊNCIA PERCEPTIVA DO RECONHECIMENTO DE LUGAR
}

\author{
Juliana Moroni ${ }^{1}$ \\ Universidade do Estado do Rio de Janeiro (UERJ) \\ https://orcid.org/0000-0002-4878-4823
}

\section{RESUMO:}

O presente trabalho está voltado à investigação epistemológica acerca da relação entre Self não conceitual, conteúdo não conceitual da percepção, prontidão para ação (awareness) ${ }^{2}$ e reconhecimento de lugar, no contexto da perspectiva ecológica gibsoniana. Tendo em vista este propósito investigativo, nossos objetivos são: 1- caracterizar o conteúdo não conceitual da percepção no contexto anti-representacionista e 2 - questionar se é necessário a consciência para os organismos agirem no ambiente e adquirirem experiências perceptivas como o reconhecimento de lugar. Argumentaremos em defesa da hipótese de que a teoria ecológico-informacional e anti-representacionista da percepção, com raízes gibsonianas, oferece subsídios teóricos inovadores para a análise do nosso propósito investigativo. Entendemos que para ter consciência e capacidade de pensar sobre si mesmo, o agente tem que, primeiramente, ter experiências perceptivas básicas do ambiente. Consideramos que essas experiências perceptivas básicas do agente, como o reconhecimento de lugar, não envolvem necessariamente a consciência.

PAlaVRaS-ChaVE: Prontidão para ação; Self não conceitual; Conteúdo não conceitual; Percepção-ação; Informação.

\section{SELF AND NONCONCEPTUAL CONTENT OF PERCEPTION: THE ANTIREPRESENTATIONIST PERSPECTIVE ON THE PERCEPTIVE EXPERIENCE OF RECOGNITION OF PLACE}

\begin{abstract}
:
The present work is focused on the epistemological investigation about the relation between Nonconceptual Self, nonconceptual content of perception, awarenes and recognition of place in the context of the Gibsonian ecological perspective. In view of this investigative purpose our objectives are the following: 1 - to characterize the nonconceptual content of perception in the anti-representationalist context and 2 - to question whether consciousness is necessary for organisms to act in the environment and to acquire perceptual experiences such as the recognition of place. In the context of Gibsonian perspective, we will argue in support of the hypothesis that the ecological-informational and anti-representationalist theory of perception offers innovative theoretical subsidies for the analysis of our research purpose. We understand that in order to have awareness and to be able to think about oneself, the agent must first have basic perceptual experiences of the environment. We consider that the agent's basic perceptual experiences, such as recognition of place, does not necessarily involve consciousness.
\end{abstract}

KEYWORDS: Awareness; Nonconceptual Self; Nonconceptual content; Perception-action; Information.

\footnotetext{
1 Doutora em Filosofia pela Universidade do Estado do Rio de Janeiro (UERJ), Rio de Janeiro - Brasil. E-mail: julianamoroni@yahoo.com.br.

${ }_{2}^{2}$ Esclarecemos que o termo awareness pode ser traduzido como senciência ou como prontidão senciente. A senciência pode ser caracterizada como a capacidade que um organismo possui de perceber/sentir conscientemente suas sensações como as de dor, por exemplo. No contexto da Filosofia ecológica, o termo senciência é usado para caracterizar a base da consciência. Para Large (2003), a consciência pode ser entendida como a associação entre awareness + percepção proprioceptiva + affordances coletivas. Neste trabalho optamos por traduzir o termo awareness como prontidão para ação de modo a dar mais ênfase significativa à concepção de percepção direta gibsoniana.
}

MORONI, Juliana. Self e conteúdo não conceitual da percepção: a perspectiva anti-representacionista acerca da experiência perceptiva do reconhecimento de lugar. Griot : Revista de Filosofia, Amargosa, Bahia, v.16, n.2, p.285-302, dezembro/2017. 285 


\section{Introdução}

Muitas teorias da percepção assumem que a percepção envolve fundamentalmente conceitos. $O$ enfoque ecológico não assume esta suposição e a trata como uma questão separada e distinta. (LARGE, 2003, p. 130, tradução nossa). ${ }^{3}$

Como caracterizar o conteúdo não conceitual da percepção? É necessário a consciência para os organismos agirem no ambiente e adquirirem experiências perceptivas como o reconhecimento de um lugar? Diversas vertentes da Filosofia da Mente e Ciências Cognitivas, pautadas no representacionismo e anti-representacionismo nos estudos da percepção, oferecem hipóteses explicativas para tais perguntas. Nosso foco de análise neste trabalho é a perspectiva anti-representacionista da percepção, relacionada aos trabalhos de Gibson (1986), Ulric Neisser (1993) e José Luis Bermudez ( 1998).

Propomo-nos a investigar o Self não conceitual, o conteúdo não conceitual e a prontidão para ação no que concerne a experiência perceptiva de reconhecimento de lugar, no contexto da percepção direta proposta originalmente por Gibson (1986). Primeiramente analisamos, com olhar crítico, a proposta ecológica não conceitual de Bermúdez (1998). Investigamos como caracterizar o conteúdo não conceitual da percepção e se a consciência é necessária para que os organismos adquiram experiências perceptivas como o reconhecimento de lugar. Concordamos com Bermúdez (1994) de que não é necessário o conceito de primeira pessoa para o reconhecimento do lugar. Porém, posteriormente, através da concepção de prontidão para ação e memória ecológica, problematizamos a posição de Bermúdez (1994) que considera necessário a consciência para o reconhecimento de lugar.

Concluímos com nossa hipótese ancorada no viés anti-representacionista gibsoniano, segundo o qual o reconhecimento de lugar pode ser feito através de estados primitivos e avançados de consciência, entretanto, tais estados não são necessários para o reconhecimento de lugar. Este pode ser realizado através da prontidão para a ação, da captação de invariantes e da memória ecológica. Nesse sentido, argumentamos que o reconhecimento de lugar ocorre através da memória ecológica, a qual caracterizamos como emergente da relação entre propriedades informacionais do ambiente e propriedades informacionais dos organismos.

\section{O conteúdo não conceitual da percepção no contexto anti-representacionista}

Existem diversas abordagens no estudo do conteúdo não conceitual da percepção na Filosofia. Há, segundo Bermúdez (2001) ao fazer menção ao trabalho de Crowther (2006), o embate entre conceitualistas puros (para os quais há estados perceptivos e conteúdos conceituais), não conceitualistas puros (para os quais há estados perceptivos e conteúdos não conceituais) e a posição intermediária entre ambas as perspectivas (para os quais há estados conceituais e conteúdo não conceitual). Conceitualistas como John McDowell negam que exista conteúdos não conceituais na experiência perceptiva. De modo geral, teóricos conceitualistas afirmam que o conteúdo representacional da experiência é sempre conceitual.

Para conceitualistas não puros, o conteúdo não conceitual da percepção pode ser caracterizado como a capacidade dos organismos de representarem a informação disponível no ambiente sem a necessidade de conceitos. Estados mentais como o de dor, por exemplo, expressam nossa experiência sem utilizar o aparato conceitual linguístico. A concepção de conteúdo não conceitual é utilizada nos estudos que explicam o comportamento dos organismos

\footnotetext{
${ }^{3}$ Many theories of perception assume that perception is fundamentally concept involving. The ecological approach does not take this as an assumption and addresses this as a separate and distinct issue. (LARGE, 2003, p. 130).
}

MORONI, Juliana. Self e conteúdo não conceitual da percepção: a perspectiva anti-representacionista acerca da experiência perceptiva do reconhecimento de lugar. Griot : Revista de Filosofia, Amargosa, Bahia, v.16, n.2, p.285-302, dezembro/2017. 286 
que não utilizam a linguagem humana para se comunicarem. Há teóricos da vertente representacionista os quais afirmam que existem diversas maneiras de representar o mundo que não são conceituais.

Para os anti-representacionistas, nosso foco de investigação neste trabalho, o conteúdo não conceitual é explicado através da percepção direta da informação disponível no ambiente, sem a necessidade de representações. Eles defendem que existem diversas maneiras de perceber diretamente a informação no mundo, sendo possível apreendê-las através de nossas atitudes não proposicionais, sem a necessidade de representar tais experiências ou recorrer a conceitos. Em ambas as vertentes, representacionista e anti-representacioniosta, questiona-se a necessidade de utilização de conceitos e proposições para se poder experienciar o mundo. Uma das questões concernentes à perspectiva anti-representacionista da percepção é explicitada da seguinte maneira: há um modo de incorporar a informação do ambiente para a ação sem que para isso seja necessário a utilização de conceitos e representações?

Em resposta a esta questão, consideramos que a proposta anti-representacionista ecológica de conteúdo não conceitual para a percepção, com base nos trabalhos de Gibson (1986) e Large (2003) mostra que sim. Antes de expormos nossa hipótese neste trabalho, analisaremos a perspectiva de Bermúdez que, num primeiro momento interage com o viés gibsoniano e, posteriormente, acrescenta um modelo de representação explícita que engloba a consciência primitiva no estudo sobre o Self e o conteúdo não conceitual.

Na perspectiva de Bermúdez (1998, p.153-154), o conteúdo não conceitual da percepção pode ser caracterizado no contexto da abordagem ecológica gibsoniana. Para ele, o conceito de percepção direta, proposto por Gibson (1986), fornece o plano básico do Self-awareness, que é a peça fundamental para a compreensão da consciência e auto-consciência. Entender como a percepção ecológica se desenvolve através da percepção da informação significativa no ambiente fornece subsídios para a compreensão das características básicas da formação do Self-awareness.

Diferentemente de outras teorias da percepção, como aquelas de cunho cartesiano, por exemplo, nas quais a representação mental funciona como mediadora entre agente percebedor e ambiente, a teoria ecológica gibsoniana propõe uma alternativa anti-representacionista para o estudo da percepção. No contexto ecológico, a percepção direta da informação não envolve inferências, proposições e representações mentais. $O$ conceito de informação ecológica é peçachave para a compreensão da percepção-ação nos moldes gibsonianos. Para Gonzalez e Morais (2007), a informação ecológica pode ser entendida como um conjunto de invariantes que possibilitam a ação. As invariantes são padrões estáveis estruturados dinamicamente através da relação de reciprocidade agente-ambiente. As invariantes, devido à sua estabilidade, são cruciais para a percepção. Como ressaltam Gonzalez e Morais (2007, p. 150):

Um dos principais recursos empregados por Gibson no estudo da percepção-ação é o conceito de informação ecológica: um conjunto de invariantes estruturais disponíveis no meio ambiente que possibilitam a realização da ação dos organismos nos seus nichos específicos. Tais invariantes, que podem ser estruturais ou de movimento, constituem affordances ou estruturas informacionais presentes no meio ambiente facilitadoras da ação.

A informação ecológica expressa-se através das affordances disponíveis no ambiente. Podemos definir resumidamente affordances como possibilidades de ação que o ambiente oferece para um organismo incorporado e situado. As affordances constituem e caracterizam o modo como o organismo vive, constituindo o seu nicho, o qual expressa o processo co-evolutivo 
organismo-ambiente. Para Gibson (1986, p. 12, tradução nossa):“Affordance implica a complementaridade do animal e do meio ambiente."4

Através da complementaridade organismo-ambiente, perceber affordance é perceber diretamente a informação significativa para a ação. Em outras palavras, para Large (2003), perceber affordances é perceber a informação do ambiente de um modo específico, direto, não inferencial. A informação ecológica emerge desse processo co-evolutivo e é captada diretamente, sem mediação de representações mentais. Para Daniela Vaz (2015, p. 147), a informação ecológica não está no organismo, em suas memórias decodificadas e nem no conhecimento inferencial, por exemplo, como expressam as teorias representacionistas da percepção. A informação ecológica está disponível no ambiente que envolve os sistemas perceptivos, sendo caracterizada como independente de qualquer processo perceptivo. A extração da informação é considerada posterior à existência da informação.

Segundo Bermúdez (1998), na teoria da percepção direta, não há distinção entre propriocepção e os cinco sentidos exteroceptivos (visão, audição, olfato, paladar e tato), uma vez que os cinco sentidos proporcionam informação proprioceptiva e exteroceptiva. Essa visão ecológica contraria a perspectiva clássica nos estudos da percepção, de acordo com a qual os cinco sentidos são exteroceptivos e exterosensitivos, os quais nos informam sobre os objetos e eventos do ambiente. Na perspectiva tradicional, a percepção do próprio corpo contrasta com a objetificação do corpo. Nesse sentido, os cinco sentidos exteroceptivos proporcionam a informação sobre o ambiente e o sistema proprioceptivo sobre o Self (postura e movimento do corpo, por exemplo). Para Bermúdez, através da concepção de affordances, cinestesia ${ }^{5}$ visual e arranjo óptico, a perspectiva ecológica oferece alternativa à visão tradicional nos estudos sobre propriocepção, exterocepção, Self e conteúdo não conceitual. Essa perspectiva expressa que temos uma prontidão para ação (awareness) contínua de nós mesmos sem necessitar de qualquer habilidade conceitual, mas apenas da co-percepção ecológica, do Self e do ambiente.

Para Bermúdez (1998, p. 154-162), a percepção direta é um processo de extrair invariantes do fluxo do arranjo óptico do ambiente. A concepção ecológica do ponto de vista não conceitual afirma, por exemplo, que os organismos percebem as invariantes que se alteram (transformacionais) e aquelas que permanecem constantes (estruturais). A interdisposição entre as invariantes permite aos organismos perceberem a informação específica que direciona a cinestesia visual. Entretanto, para Bermúdez, isso só é possível através da copercepção ecológica entre Self e ambiente, mais a capacidade de reconhecimento do lugar. Mas, qual a importância da capacidade de reconhecimento do lugar? No que concerne a essa pergunta, Bermúdez sustenta que (1998, p. 163):

1- O reconhecimento do lugar está relacionado ao reconhecimento de que objetos existem independentemente de serem experienciados por um organismo;

2- Ao reconhecer determinado lugar, um organismo adquire prontidão para a ação (awareness) e a percepção de que o lugar já existia no passado e de que ele já esteve neste lugar (have been there). Ao reconhecer o lugar e perceber que já esteve neste lugar, o organismo necessita de estados de consciência;

3- A capacidade de reconhecimento do lugar está associada ao senso de identidade transtemporal dos lugares e do Self.

\footnotetext{
${ }^{4}$ It implies the complementarity of the animal and the environment. (GIBSON, 1986, p. 127).

${ }^{5}$ De modo geral, a cinestesia é a percepção dos próprios movimentos corporais no ambiente. Já a sinestesia é a relação entre planos sensoriais diferentes, por exemplo, a visão com o cheiro, quando alguém vê a foto de outra pessoa e sente seu cheiro, ou da relação entre visão e audição, quando alguém vê as cores da música que está ouvindo. Neste trahalho, o nosso foco de estudo é a cinestesia no contexto da Filosofia Ecológica. (Fonte: http://pt.wikipedia.org/wiki/Sinestesia). Para uma compreensão mais detalhada da sinestesia ver o artigo How do synesthetes experience the world?, escrito por Ophelia Deroy.
}

MORONI, Juliana. Self e conteúdo não conceitual da percepção: a perspectiva anti-representacionista acerca da experiência perceptiva do reconhecimento de lugar. Griot : Revista de Filosofia, Amargosa, Bahia, v.16, n.2, p.285-302, dezembro/2017. 288 
Como veremos nos parágrafos seguintes, diferentemente de Bermúdez que pressupõe a necessidade de um estado de consciência primitivo para experiências perceptivas como o reconhecimento de lugar, consideramos que o Self não conceitual, prioriza as sensações corpóreas em detrimento às concepções que definem a identidade pessoal exclusivamente pelo cérebro, por um Self reflexivo e consciente. A prioridade das sensações corpóreas expressa o Self não conceitual como incorporado e situado, dependente do tipo de experiências que o corpo vivencia, no que concerne às suas capacidades sensório-motoras, no contexto físico, biológico e social. (GONZALEZ; HASELAGER, 2003, p. 99)

Como mostraremos, aceitamos que o reconhecimento do lugar possa ser feito através de estados primitivos de consciência e da percepção que envolve inferências. Entretanto, argumentamos que nem a consciência e tampouco a percepção inferencial são condições sine qua non para o reconhecimento do lugar. Nas palavras de Large (2003, traduzido por MORONI; GONZALEZ; MORAES, 2011, p.353):

Isto não equivale negar que o raciocínio pode envolver etapas ou que inferimos algo de algo. Significa apenas que a experiência consciente produz conhecimento em um modo que não é o produto da razão sozinha; e também que o conhecimento não pode ser o resultado de etapas computacionais desempenhadas no interior do perceptor.

Para Bermúdez, o Self pode ter compreensão (grasp) de modo não conceitual através da representação proto-intencional do comportamento. Ele sugere uma forma de representação não conceitual do Self. Para isso, ele utiliza a co-percepção do Self e ambiente proposta por Gibson (1986), incluindo nesta concepção a capacidade dos organismos de reconhecerem um lugar e representarem a si mesmos num plano não conceitual.

Para Bermúdez (1998), o reconhecimento de lugar envolve o registro senciente e consciente de que determinado organismo tinha estado naquele lugar específico. As críticas de Bermúdez à concepção ecológica gibsoniana estão relacionadas à insuficiência da proposta ecológica para explicar o reconhecimento do lugar apenas pela percepção direta de invariantes no ambiente. Segundo Large (2003), as invariantes são caracterizadas como propriedades de padrões informacionais que permanecem constantes e que determinam o que está disponível para a percepção durante as mudanças no sistema organismo-ambiente. Elas proporcionam estabilidade ao ambiente, propiciando estrutura informacional adequada para cada contexto em que vivem os organismos. Nesse contexto, as invariantes também são caracterizadas como padrões informacionais coletivos que emergem da relação entre agente e ambiente. Para Heft (2007, p. 95), as invariantes também estão relacionadas à "construção do nicho", na medida em que o ambiente carrega a marca das atividades dos organismos na sua relação de reciprocidade com o ambiente. Essas marcas são indícios de que os organismos desempenharam atividades a fim de se adaptarem ao lugar em que vivem.

Nesse sentido, para Bermúdez, no que concerne ao reconhecimento de lugar, a percepção ecológica não envolve memória consciente. Ele cita o exemplo do pássaro que se desloca para vários lugares diferentes, mas não reconhece conscientemente os lugares por onde voa repetidas vezes. Isso porque o pássaro apenas tem a prontidão para ação (awareness) do movimento através do espaço-tempo. O presente emerge de modo contínuo e se estende em direção a um ponto de vista temporalmente estendido. Nesse sentido, o ponto de vista é construído na estrutura da percepção. Para explicar o reconhecimento de lugar em relação ao pássaro, por exemplo, podemos dizer que, no viés de Gibson, os sistemas perceptivos do pássaro ficam mais suscetíveis e sensíveis as formas particulares de informação através do tempo. Para Bermúdez, essa explicação de cunho gibsoniano é insuficiente, dado que o reconhecimento do lugar requer o senso de identidade transtemporal dos lugares e a identidade transtemporal do Self. Ele mescla a

MORONI, Juliana. Self e conteúdo não conceitual da percepção: a perspectiva anti-representacionista acerca da experiência perceptiva do reconhecimento de lugar. Griot : Revista de Filosofia, Amargosa, Bahia, v.16, n.2, p.285-302, dezembro/2017. 289 
copercepção do Self e do ambiente ao reconhecimento consciente do lugar. Como ressalta Bermúdez (1998, p. 172, tradução nossa):

Nós começamos com a co-percepção básica do Self e do ambiente descrita pela proposta ecológica da estrutura da percepção de Gibson e a ampliamos ao argumentar que criaturas para as quais é legítimo atribuir um ponto de vista devem ser capazes de reconhecimento consciente do lugar e de representar a si mesmas de uma maneira explícita. ${ }^{6}$

De acordo com o excerto acima, a consciência não conceitual necessita de algum tipo de representação de si explícita não conceitual. Podemos, no entanto, questionar: 1- $O$ que seria essa representação explícita não conceitual? 2- $\mathrm{O}$ reconhecimento do lugar envolve a consciência no seu modo primitivo como sugere Bermúdez?

A representação não conceitual e explícita ligada ao reconhecimento de lugar e de objetos, segundo Bermúdez (1998), está atrelada ao comportamento proto-intencional dos organismos. Ela vai além da capacidade estímulo-resposta $(E-R)$ proposta pela psicologia behaviorista. Para exemplificar este tipo de representação explícita, consciente e não conceitual, Bermúdez se refere a testes com crianças de 3 meses, as quais observavam um objeto suspenso acima dos seus berços. $\mathrm{O}$ objeto se movia de diferentes modos e as crianças o acompanhavam com diferentes tipos de reações de prazer. Algumas vezes elas apenas observavam e outras elas interagiam com o objeto. Ao interagirem com o objeto, demonstravam comportamento de maior deleite. Segundo Bermúdez, o comportamento de interação dessas crianças com o respectivo objeto não pode ser explicado através de representações mentais e nem somente pela concepção ecológica de percepção direta e prontidão para a ação (awareness). Bermúdez oferece uma terceira via para explicar o teste, recorrendo às representações explícitas não conceituais; as crianças sentem prazer ao interagir com os objetos, porque estão cientes (aware) do que estão fazendo, estão cientes de agir sobre coisas que são diferentes delas e de prontidão para agirem. Para ele, elas têm consciência da distinção entre suas próprias intenções ou atos e dos movimentos do objeto no ambiente. Assim, o Self pode ser representado através do raciocínio espacial, sem necessariamente ser preciso o conceito de primeira pessoa.

Entretanto, como questionamos em 2, o reconhecimento do lugar envolve um self consciente e sujeito à representações explícitas de si mesmo? Como sugere Bermúdez, a consciência é necessária para o reconhecimento do lugar devido ao fato de que a concepção ecológica gibsoniana não é suficiente para explicar como, no plano não conceitual, o reconhecimento de lugar ocorre através da percepção direta:

O ponto é que, tal como se apresenta, a concepção gibsoniana não pode fazer todo o trabalho que foi sugerido a ela. A sugestão era que a concepção gibsoniana de percepção ecológica pudesse mostrar como algo como um ponto de vista não conceitual se reflete na estrutura da percepção. Parece-nos agora que, entretanto, isso não será obtido até que a capacidade consciente de reconhecimento de lugar seja adicionada à copercepção ecológica do Self e do ambiente. (BERMÚDEZ, 1998, p. 163, tradução nossa) ${ }^{7}$

\footnotetext{
${ }^{6}$ We started with the basic coperception of self and environment described in Gibson's ecological account of the structure of perception and built it up by arguing that creatures to which it is legitimate to ascribe a point of view should be capable of conscious place recognition and of representing themselves in an explicit manner. (BERMÚDEZ, 1998, p. 172).

7 The point is that, as it stands, the Gibsonian account cannot do all the work it was suggested it might be able to do. The suggestion was that the Gibsonian account of ecological perception could show how something like a nonconceptual point of view is reflected in the very structure of perception. It now seems, however, that this will not be achieved until the appropriate capacity for conscious place recognition is added to the ecological coperception of self and environment. (BERMÚDEZ, 1998, $163)$.
}

MORONI, Juliana. Self e conteúdo não conceitual da percepção: a perspectiva anti-representacionista acerca da experiência perceptiva do reconhecimento de lugar. Griot : Revista de Filosofia, Amargosa, Bahia, v.16, n.2, p.285-302, dezembro/2017. 290 
Em resposta à esta afirmação de Bermúdez, no excerto acima, podemos argumentar que o Self não conceitual se caracteriza como um rede de relações e um processo gerador e detector de padrões emocionais através de experiências perceptivas básicas. Nesse sentido, questionamos se o reconhecimento de lugar necessita mesmo de consciência. Em resposta a essa pergunta é necessário entender a diferença entre consciência (consciouness) e prontidão para a ação (awareness). Essa diferença pode ser expressa na seguinte passagem de Large ((2003, p. 74, tradução nossa):

Há, na percepção, uma distinção entre consciência e prontidão para ação, eu posso, por exemplo, estar consciente de perceber a Mona Lisa enquanto eu estou tendo prontidão para ação das paredes do Louvre e não estar consciente de percebê-las. Considera-se que certos animais como cachorros e pássaros têm prontidão para ação, mas não são perceptivamente conscientes. ${ }^{8}$

Concordamos com a visão de Large acerca da diferença entre prontidão para a ação e consciência. Entretanto, ao expressar essa diferença, consideramos que ele comete um equívoco ao afirmar que cães e pássaros não são caracterizados como seres com consciência. Argumentamos que, nesta passagem, Large expressa uma visão antropocêntrica, a qual se choca com a perspectiva não antropocêntrica da Filosofia Ecológica, causando uma contradição no interior da própria teoria que Large defende. Isso porque há várias pesquisas em neurociência e em biologia sobre estudos da consciência em humanos e outros animais, as quais resultaram, por exemplo, no manifesto (2012) da consciência assinado por Philip Low, David Edelman, Christof Koch, Stephen Hawking, entre outros. Segundo os autores do manifesto:

\begin{abstract}
Nós declaramos o seguinte: a ausência do neocortex não parece impedir um organismo de experienciar estados afetivos. Evidências convergentes indicam que animais nãohumanos possuem substratos neuroanatômicos, neuroquímicos e neurofisiológicos dos estados conscientes, juntamente com a capacidade de exibir comportamentos intencionais. Consequentemente, o peso da evidência indica que os humanos não são únicos a possuírem os substratos neurológicos que geram consciência. Animais nãohumanos, incluindo todos os mamíferos e aves, e muitas outras criaturas, incluindo polvos, também possuem esses substratos neurológicos ${ }^{9}$
\end{abstract}

Ainda, outros estudos constatam que as abelhas podem memorizar e reconhecer faces. ${ }^{10}$ Com isso queremos mostrar que mesmo as pesquisas realizadas num viés representacionista expressam que a visão antropocêntrica de que somente humanos possuem consciência é equivocada. ${ }^{11}$

\footnotetext{
8 Within perception there is a distinction between consciousness and awareness. I may for instance be conscious of perceiving the Mona Lisa whereas I may be aware of perceiving the walls of the Louvre and not be conscious of perceiving them. It is taken that certain animals such dogs and birds are perceptually aware but not perceptually conscious. (LARGE, 2003, p. 74).

9 We declare the following: "The absence of a neocortex does not appear to preclude an organism from experiencing affective states. Convergent evidence indicates that non-human animals have the neuroanatomical, neurochemical, and neurophysiological substrates of conscious states along with the capacity to exhibit intentional behaviors. Consequently, the weight of evidence indicates that humans are not unique in possessing the neurological substrates that generate consciousness. Nonhuman animals, including all mammals and birds, and many other creatures, including octopuses, also possess these neurological substrates. (LOW; EDELMAN; KOCH; HAW KING, 2012).

${ }^{10}$ (Fonte:http://www.scientificamerican.com/article/face-recognition-honeybees/e http:/www.scientificamerican.com/article/insects-recognize-faces-using-processing-mechanism-similar-to-that-of-humans/).

11 Com relação à memória, estudos recentes mostram que insetos assim como outros organismos podem desenvolver falsas memórias. Pesquisadores dispuseram três flores artificiais: uma amarela, outra preta com anéis brancos e ainda uma terceira com anéis amarelos, uma mistura dos padrões das duas primeiras. As abelhas foram treinadas para encontrar água com açúcar nestas flores. As abelhas mostraram preferência pelas pétalas de flores que continham açúcar. Depois de alguns dias, as abelhas começaram a selecionar a flor com o anel amarelo mesmo que inicialmente não proporcionassem nada a elas. Os pesquisadores afirmam que era como se suas memórias tivessem se misturado, ou seja, as abelhas tiveram memórias falsas como os humanos
}

MORONI, Juliana. Self e conteúdo não conceitual da percepção: a perspectiva anti-representacionista acerca da experiência perceptiva do reconhecimento de lugar. Griot : Revista de Filosofia, Amargosa, Bahia, v.16, n.2, p.285-302, dezembro/2017. 291 
Porém, como veremos no próximo tópico, no nosso trabalho procuramos mostrar que a memória associada à capacidade de reconhecimento do lugar pode ser explicada num viés antirepresentacionista, o qual não envolve necessariamente a consciência, mas apenas a prontidão para a ação.

\section{Memória ecológica: o reconhecimento de lugar via percepção direta}

"Autopercepção e percepção do ambiente caminham juntos." (GIBSON, 1986, p. 116, tradução nossa). ${ }^{12}$

A experiência perceptiva de reconhecimento de lugar pode emergir diretamente, através da prontidão para a ação e da memória ecológica, sem envolver necessariamente a consciência. Para deixarmos claro essa hipótese de nosso trabalho, consideramos pertinente esclarecer alguns conceitos constitutivos da perspectiva ecológica da percepção a fim de caracterizarmos a memória ecológica, tais como: Self não conceitual, campo de visão, mundo visual, exterocepção, propriocepção, ressonância, prontidão para a ação, percepção direta, invariantes, affordances e os padrões que as ligam.

De acordo com Neisser (1993), foi Gibson quem primeiramente sugeriu que Self e ambiente estão atrelados de tal forma que perceber o Self é perceber o ambiente e vice-versa. Nas palavras de Gibson (1986, p. 126, tradução nossa):

Informação sobre o Self acompanha informação sobre o ambiente, e os dois são inseparáveis. Autopercepção acompanha exterocepção como o outro lado de uma moeda. A percepção tem dois polos, o subjetivo e o objetivo, e a informação está disponível para especificar ambos. Percebemos o ambiente e copercebemos a nós mesmos. (GIBSON, 1986, p. 126). ${ }^{13}$

Nos estudos sobre a percepção e o Self não conceitual, a abordagem ecológicainformacional e anti-representacionista gibsoniana expressa um deslocamento de foco do cérebro para a relação corpo-ambiente. A proposta dessa abordagem é entender não o que há dentro de nossa cabeça, mas dentro do que está nossa cabeça. Como ressalta Large (2003, p. 130.):

A abordagem ecológica assegura que focalizando no cérebro e no que acontece no "interior", por exemplo, em nossa psique, muitas explicações têm sido direcionadas a uma concepção de mente e consciência que é muito limitada. Aceitar a abordagem ecológica equivale a renunciar à ideia de que a mente é algo na cabeça com sua causa fora de si mesma. Você deveria, como o slogan diz, perguntar não o que está dentro de sua cabeça, mas dentro do que está a sua cabeça. Assim a abordagem ecológica oferece insights libertadores na natureza da mente, libertando-nos, por exemplo, das garras da concepção mecanicista da mente. (LARGE, 2003, p.130, traduzido por MORONI; GONZALEZ; MORAES, 2011, p. 353-354). ${ }^{14}$

\footnotetext{
em situações em que esquecem algo em um lugar e procurar em outro. (Fonte: http://www.scientificamerican.com/podcast/episode/animals-can-be-given-false-memories/).

12 Self-perception and environment perception go together. (GIBSON, 1986, p. 116).

13 "Information about the self accompanies information about the environment, and the two are inseparable. Egoreception accompanies exteroception, like the other side of a coin. Perception has two poles, the subjective and the objective, and information is available to specify both. One perceives the environment and coperceives oneself." (GIBSON, 1986, p. 126).

14 The ecological approach holds that by focusing on the brain and what goes on 'inside', e.g. in our psyche, many previous accounts have been led into a conception of mind and consciousness that is too narrow. To accept the ecological approach is to relinquish the idea that the mind is something in the head with its cause outside of itself. You should, as the slogan goes, ask not what's inside your head but what your head's inside. Thus the ecological approach offers liberating insights into the nature of mind, freeing us, for example, from the grip of the mechanistic picture of the mind. (LARGE, 2003, p.4).
}

MORONI, Juliana. Self e conteúdo não conceitual da percepção: a perspectiva anti-representacionista acerca da experiência perceptiva do reconhecimento de lugar. Griot : Revista de Filosofia, Amargosa, Bahia, v.16, n.2, p.285-302, dezembro/2017. 292 
Nesse sentido, questionar, apoiados em Gibson, "dentro do que está nossa cabeça" direciona nossas investigações para uma abordagem não mecanicista nos estudos em Filosofia da Mente, a qual leva em consideração a relação de reciprocidade organismo-ambiente via percepção direta. Assim, podemos afirmar que o estudo do Self não conceitual está direcionado para as experiências corpóreas em detrimento ao Self consciente no plano conceitual.

Para Eleonor Gibson (1993, p. 35-37), o Self se relaciona com o ambiente de modo recíproco. As dimensões do Self são percebidas em relação às dimensões do ambiente e as dimensões e propriedades do ambiente limitam as ações do organismo. É a partir da interação entre propriedades do ambiente e propriedades do corpo que emerge o conhecimento de Nós mesmos através de nossa percepção-ação. Para Neisser (1993), a partir dessa interação percepção-ambiente emergem cinco tipos básicos de informações sobre Nós mesmos, ou como ele denominou, auto-conhecimento. Cada informação especifica um aspecto diferente do indivíduo, bem como expressa diferentes tipos de Selves, os quais são denominados Self ecológico (ecological Self), Self interpessoal (interpersonal Self), Self conceitual (conceptual Self), Self particular (particular Self) e Self ampliado (extended Self). Nos interessa neste trabalho o Self ecológico e o Self interpessoal dado que são constituintes do Self nao conceitual.

Neisser (1993, p.4-5) caracteriza o Self ecológico da seguinte maneira: é o indivíduo considerado como um agente ativo no ambiente imediato da percepção. Este agente percebe a si mesmo e outras coisas no ambiente físico, por exemplo, onde estamos, o que estamos fazendo, se efetuamos uma determinada ação, o que deveríamos fazer, etc. De acordo com Neisser (1993, p. 5-11), o Self ecológico não pode ser reduzido ao corpo biológico. Nós temos a prontidão para ação (awareness) e percebemos os efeitos dessas ações nos nossos movimentos. A informação possibilita a percepção dos nossos movimentos e de sua direcionalidade. Existe uma resposta, um feedback cinestésico dos músculos e das articulações, bem como a detecção da aceleração de órgãos vestibulares. Os cinco sentidos são importantes para o Self ecológico, por exemplo, o tato ou a informação acústica que alguns animais como o ser humano com cegueira e o morcego usam para se locomoverem no ambiente. Mas o foco gibsoniano é a visão.

Já o Self interpessoal é o mesmo indivíduo caracterizado como Self ecológico em outro contexto. Nesse contexto, há interação face a face com outros indivíduos, os quais são percebidos, assim como no Self ecológico, através dos movimentos, das posições do corpo do indivíduo e das emoções. Para Neisser, essas duas formas de selves não são conceitualizadas, lembradas, imaginadas ou construídas, mas experienciadas pelos agentes.

Para Neisser (1993, p. 10-17), como ressaltamos, o Self ecológico é caracterizado como o agente ativo no ambiente físico; já o Self interpessoal é o agente envolvido numa interação social, não reflexiva com outras pessoas. Numa atividade comunicativa entre grupos culturalmente diferentes, por exemplo, cada membro desses grupos é considerado um Self interpessoal. Numa relação entre duas pessoas, por exemplo, são estabelecidos diversos padrões recíprocos, objetivos e perceptivos que expressam estados emocionais, desejos, etc que afirmam a interação, mutualidade, as diferenças e a individualidade do Self interpessoal e da identidade pessoal. ${ }^{15}$

Nesse sentido, segundo Neisser (1993), o Self interpessoal também é um agente ativo no ambiente. Entretanto, a diferença entre o Self ecológico e o Self interpessoal reside no seguinte aspecto: a informação que é produzida pelo movimento que caracteriza a ação do Self ecológico está alicerçada em princípios ecológicos universais, os quais são considerados os mesmos para animais humanos e não humanos. Já a informação produzida pela ação e comportamento do Self interpessoal é considerada específica para cada espécie. Por exemplo, como assinala Neisser, gatos não apertam as mãos e as aranhas não valorizam o contato olho no olho como o fazem os

15 Não é nosso objetivo neste trabalho estudar a identidade pessoal.

MORONI, Juliana. Self e conteúdo não conceitual da percepção: a perspectiva anti-representacionista acerca da experiência perceptiva do reconhecimento de lugar. Griot : Revista de Filosofia, Amargosa, Bahia, v.16, n.2, p.285-302, dezembro/2017. 293 
humanos. Baseados na informação presente na estrutura óptica do ambiente e na informação específica entre espécies, ambos, Self ecológico e Self interpessoal, são agentes de comunicação não-verbal. Como afirma Neisser (1988, p. 393, tradução nossa):

Assim como o Self ecológico é especificado pela orientação e pelo fluxo da textura ótica, é o Self interpessoal especificado pela orientação e fluxo dos gestos expressivos de outro indivíduo; assim como o Self ecológico é articulado e confirmado pelos efeitos de nossas próprias ações físicas, é o Self interpessoal desenvolvido e confirmado pelos efeitos de nossos próprios gestos expressivos sobre nossos parceiros. ${ }^{16}$

Assim, pode-se questionar: a percepção ecológica e a percepção social originam-se independentemente uma da outra? Neisser (1993) afirma que tanto o Self social como o não social são originados independentemente. Sem a contribuição de experiências específicas do Self interpessoal, algumas formas de conhecimento não poderiam existir. Para ele, a percepção social não pode ser reduzida à percepção ecológica, assim como o Self interpessoal não pode ser reduzido ao Self ecológico.

A emergência do Self não conceitual está intrinsecamente relacionada à percepção direta e ao arranjo do ambiente (ambient array), bem como a informação sobre o Self está interligada à informação sobre o ambiente. Assim, ao perceber o ambiente, o organismo percebe a si mesmo. No caso da percepção visual, de acordo com Gibson (1986, p. 111-126), o campo de visão do organismo é mais limitado se comparado com o arranjo do ambiente. É apenas uma parte, um modelo do ambiente como todo. $\mathrm{O}$ âmbito angular do campo de visão depende de como os olhos estão posicionados na cabeça. Cada sistema ocular propicia um campo de visão, os cavalos, por exemplo, tem olhos laterias. A mescla desses dois sistemas proporciona ao cavalo um campo de visão semipanorâmico. Já os seres humanos, por exemplo, têm olhos frontais e cada um dos sistemas oculares propicia um campo de visão hemisférico. A junção desses dois sistemas oculares fornecem aos humanos um campo de visão mais amplo do que o semipanorâmico dos cavalos.

De acordo com Gibson (1986, p. 206-207), a diferença entre o campo de visão (visual field) e mundo visual (visual world) se apresenta, por exemplo, no fato de o campo visual ter limites que são facilmente observados por um perceptor atento, como possuir a parte central evidente e a periférica indefinida, enquanto o mundo visual não tem limites, como a superfície de uma esfera que se estenda diante de um organismo que não possui centro de definição, dado que é evidente por todos os lados. O campo de visão emerge dos olhos fixos e está relacionado ao olhar para um objeto específico (looking at). É o movimento dos olhos relativo à cabeça e a um determinado objeto. Já o mundo visual emerge do ambiente quando determinado organismo olha ao redor e com os dois olhos de diferentes pontos de observação; ele está relacionado ao olhar ao redor, no que se refere a uma visão panorâmica (looking around). Como ressalta Gibson (1986, p. 205, tradução nossa): "Vê-se o ambiente não com os olhos, mas com os olhos-na cabeça-no-corpo-que repousa-no-chão." 17 O mundo visual é o resultado da percepção da informação invariante no arranjo óptico do ambiente, através da exploração do sistema visual e da prontidão para a ação (awareness) do organismo em relação ao seu próprio corpo no mundo. $\mathrm{O}$ campo visual e o mundo visual contribuem para a emergência da memória ecológica, através do

\footnotetext{
${ }^{16}$ Just as the ecological self is specified by the orientation and flow of optical texture, so the interpersonal self is specified by the orientation and flow of other individual's expressive gestures; just as the ecological self is articulated and confirmed by the effects of our own physical actions, so the interpersonal self is developed and confirmed by the effects of our own expressive gestures on our partner. (NEISSER, 1988, p. 393.)

17 One sees the environment not with the eyes but with the eyes-in-the-head-on-the-body-resting-on-the ground. (GIBSON, 1986, p. 205).
}

MORONI, Juliana. Self e conteúdo não conceitual da percepção: a perspectiva anti-representacionista acerca da experiência perceptiva do reconhecimento de lugar. Griot : Revista de Filosofia, Amargosa, Bahia, v.16, n.2, p.285-302, dezembro/2017. 294 
sistema perceptivo visual. No caso de outros sistemas perceptivos, como o relacionado ao tato, por exemplo, a percepção ocorre através do toque e envolve a detecção da informação invariante das superfícies e da prontidão para a ação do organismo em relação ao seu corpo no ambiente. A percepção está relacionada à propriocepção e à exterocepção.

Segundo Gibson (1986), a propriocepção é considerada egorecepção (egore-ception) dado que está relacionada ao Self, mas não somente como um canal específico de sensações. A egorocepção proporciona informações múltiplas ao Self na sua relação de reciprocidade contínua com o ambiente. Como exemplo dessas múltiplas informações podemos pensar que um indivíduo vê a si mesmo, ouve seus passos, sente seus músculos dobrarem, ouve sua voz, sente a sua mão e pele ao mesmo tempo quando toca a sua pele, etc. Nesse sentido, a informação óptica que especifica o Self para a auto-percepção (cabeça, corpo, braços, mãos, etc) é inseparável da informação que especifica o ambiente para a percepção do ambiente. Essa inseparabilidade pode ser descrita quando um homem vê o ambiente e seu nariz ao mesmo tempo. Como ressalta Gibson (1986, p. 116, tradução nossa): "Quando um homem vê o mundo, ele vê seu nariz ao mesmo tempo." 18

Nessa passagem, Gibson mostra que há diferentes tipos de gradientes de diferentes texturas e densidades para a percepção, neste caso, visual. No que concerne ao exemplo do nariz e do mundo, há um layout contínuo de superfícies que se estende entre o nariz e o mundo. Os gradientes de percepção especificam o layout do ambiente e a distância entre o nariz do observador e o horizonte, expressando a complementaridade entre propriocepção e exterocepção. Essa complementaridade pode ser observada através da cinestesia que é caracterizada como o senso do movimento (músculos e articulações) que temos do nosso corpo ao pedalar uma bicicleta, caminhar, correr, jogar futebol, etc.

Neste contexto, a pergunta que colocamos é a seguinte: em que medida os gradientes para a percepção estariam relacionados ao reconhecimento de lugar num viés antirepresentacionista sem envolver a consciência (como sugere Bermúdez)? Em resposta a esta pergunta e à pergunta anterior - se na concepção ecológica anti-representacionista com raíz gibsoniana, o reconhecimento de lugar necessita da consciência -, sustentamos que o reconhecimento de lugar, num primeiro momento, não envolve consciência. Isso porque a memória ecológica está associada à percepção direta dos organismos na sua coevolução com o ambiente, sem a necessidade de possuir níveis mais elevados de percepção que envolvam a consciência ou a auto-consciência, mesmo que num plano não conceitual, como defende Bermúdez.

Como argumentamos, o reconhecimento de lugar pode ser feito através da memória ecológica ${ }^{19}$ que emerge da relação entre as propriedades informacionais do ambiente e aquelas encontradas no corpo incorporado e situado. Para Gibson (1986), o fluxo da experiência não consiste do momento presente instantâneo e tampouco do passado linear. Não podemos perceber em que momento a percepção pára e em que instante ela se volta ao passado através de

\footnotetext{
18 When a man sees the world, he sees his nose at the same time. (GIBSON, 1986, p. 116).

19 A memória ecológica difere da memória no sentido tradicional, na medida em que a primeira não necessita de armazenamento de representações de eventos para direcionar o input e especificar o objeto, lugar ou evento do ambiente e fazer emergir a memória correta. Na concepção tradicional, a memória apresenta a seguinte falha: se a memória é um conjunto de representações de eventos, objetos, lugares do ambiente armazenados no cérebro, o problema é entender como o input específica a representação do evento que será lembrado? Se o objeto tem de ser especificado com precisão para se acessar a memória correta, o conceito de armazenamento de representações se torna desnecessário. (MICHAELS\&CARELLO, 1981, p. 185-186). Assim, se a memória não representa corretamente a experiência, o padrão de reconhecimento é um problema para a teoria tradicional da memória. Esse problema desaparece na concepção ecológica de memória, na medida em que ela não necessita de armazenamento de representações, mas dos padrões de reconhecimento que especificam diretamente a memória através da captação da informação dos eventos, objetos e lugares do ambiente. O conceito de memória será estudado com mais precisão em trabalhos posteriores.
}

MORONI, Juliana. Self e conteúdo não conceitual da percepção: a perspectiva anti-representacionista acerca da experiência perceptiva do reconhecimento de lugar. Griot : Revista de Filosofia, Amargosa, Bahia, v.16, n.2, p.285-302, dezembro/2017. 295 
lembranças. Não existe linha divisória entre presente e passado, perceber é lembrar na medida em que a percepção é contínua: "Uma percepção não tem um fim. A percepção continua." (GIBSON, 1986, p. 253, tradução nossa). ${ }^{20}$ Para ele, a teoria da captação da informação (pick up information) não precisa do auxílio da memória entendida como ponte na divisão passado e presente. Essa compreensão de memória clássica ${ }^{21}$ pode influenciar a captação de invariantes e affordances no ambiente, mas não é crucial para a percepção.

Nesse sentido, podemos caracterizar a memória ecológica da seguinte forma: o sistema perceptivo, neste caso visual ${ }^{22}$ entra em contato com as invariantes e affordances disponíveis no ambiente através do olhar para (looking at) e do olhar ao redor (looking around), captando diretamente a informação e promovendo ajustes a fim de que o organismo mantenha integradas as suas experiências perceptivas de modo contínuo, não linear e sem fragmentações. Passado e presente se fundem nas trocas de propriedades informacionais (invariantes) entre organismos e ambiente. A combinação dessas propriedades possibilita a emergência das affordances que ocorrem no domínio das regularidades que perpassam a relação entre percepção-ação. A fusão das propriedades relacionadas à conexão corpo-ambiente envolvem os diversos tipos de gradientes para a percepção ${ }^{23}$, também envolvem a prontidão para ação (awareness) e a ressonância para a percepção dos padrões de ajustes relacionados à informação significativa.

Segundo Gibson (1986, p. 183-184), a prontidão para a ação de locomoção é caracterizada pela percepção visual da própria locomoção no ambiente. A cinestesia visual registra tanto movimentos do corpo quanto do sistema muscular que é interno ao organismo. A visão capta tanto os movimentos do corpo como um todo relativo à superfície, quanto os movimentos de partes do corpo relativos ao todo corporal. Isso expressa, na visão ecológica gibsoniana, que é um equívoco considerar que a percepção visual é apenas exteroceptiva, ou seja, que ela capta somente a informação externa do ambiente. Para Gibson, ela é tanto exteroceptiva quanto proprioceptiva, pois relaciona as informações do corpo com as informações do ambiente, numa relação de mutualidade. Como ressalta Large ( 2003 , p. 126, tradução nossa) "No enfoque ecológico, o que é sentido (percebido) é o fluxo ótico. Este pode ser descrito como um fluxo ao redor do perceptor governado pelas leis da perspectiva do movimento. O perceptor tem prontidão para ação do fluxo óptico, mas não é consciente dele."24

De acordo com Large (2003, p. 123), há, na teoria gibsoniana uma mudança de foco da teoria da sensação para a percepção para a teoria da informação para a percepção, ou seja, ela se baseia na informação em detrimento da sensação, o que propiciou novas implicações nas concepções de conhecimento, reconhecimento de lugar, memória, significado e Self. Mas quais as

\footnotetext{
${ }^{20}$ A perception does not have an end. Perceiving goes on.(GIBSON, 1986, p. 253).

21 Segundo Broens $(2001$, p. 32), para a concepção clássica cartesiana, há dois tipos de memória: a memória mecânica e a memória ligada ao intelecto. A primeira está associada ao "controle do movimento corporal e à capacidade de recuperar voluntariamente as representações intelectuais impressas no cérebro." Já a memória intelectual está associada "a todas as atividades cognitivas exercidas metodicamente pela substância pensante[...]". Broens sugere que a memória de cunho cartesiano pode ser considerada tanto atividade corpórea quanto atividade do intelecto, "ora explicada como possibilidade mecânica de preservar representações intelectuais e repetir gestos, ora simplesmente não explicada [...]". Não é nosso propósito neste trabalho efetuar um estudo da concepção de memória cartesiana.

22 No que concerne a relação entre outros sistemas perceptivos como tato e olfato, por exemplo, consideramos que a definição de memória ecológica também é aplicada. Isso através da troca de informações e ajustes entre as propriedades do acoplamento organismo-ambiente. Essa troca de propriedades envolve, como mencionamos no texto, prontidão para ação e ressonância. Não é o objetivo deste trabalho estudar outros tipos de sistemas perceptivos e suas características peculiares ligadas à concepção de memória ecológica.

23 Neste trabalho, a expressão "gradientes para a percepção" é definida como a variação gradativa de propriedades e características do ambiente, como os gradientes de textura do solo e das árvores, por exemplo, para a percepção direta.

24 On the ecological approach what is sensed is the optic flow. This can be described as a flow surrounding the perceiver governed by the laws of motion perspective. The perceiver is aware of the optic flow but not conscious of it. (LARGE, 2003, p. 126).
}

MORONI, Juliana. Self e conteúdo não conceitual da percepção: a perspectiva anti-representacionista acerca da experiência perceptiva do reconhecimento de lugar. Griot : Revista de Filosofia, Amargosa, Bahia, v.16, n.2, p.285-302, dezembro/2017. 296 
diferenças cruciais no papel da teoria da sensação para a percepção e da informação para a percepção? A primeira caracteriza-se por ser representacionista e adota o realismo indireto. Entre suas principais particularidades está a concepção de que há intermediários entre o organismo e o ambiente. Esses intermediários seriam as representações, ideias, proposições, interpretações, inferências, etc. Além disso, a teoria indireta da sensação para a percepção caracteriza-se como um processo com etapas múltiplas. Esse processo inicia-se quando as energias físicas encontram a superfície sensorial, a qual propicia a emergência das sensações. Essas sensações são transformadas através de etapas processuais e operações cognitivas até atingir o ponto final, que é o objeto da percepção do ambiente. $O$ perceptor processa os inputs sensoriais adquiridos do ambiente, unindo-os a outros dados sensoriais, alguns armazenados na memória, para realizar o output sensorial e a ação.

A informação para a percepção, por sua vez, é caracterizada, segundo Large (2003-p. 121123), como anti-representacionista e pertencente à vertente do realismo direto com raízes gibsonianas. Entre suas principais peculiaridades estão: 1- Os objetos físicos são percebidos diretamente e 2- A percepção dos objetos físicos não envolve representação, interpretação, inferências, processamento de informação, etc. Nesse sentido, a teoria da informação para a percepção defende que a percepção é direta e que não necessita de processamento sensorial para proporcionar ação ao organismo. A percepção visual, por exemplo, é resultado da captação da informação presente nas estruturas invariantes. Essas estruturas especificam o layout do ambiente e qualquer animal que seja sensível a essa estrutura, devido à sua história filogenética e ontogenética, é capaz de captar a informação de modo direto. Atividades do organismo como o movimento dos olhos ou da cabeça, por exemplo, proporcionam mudanças no arranjo do ambiente e facilita a detecção das invariantes em tal estrutura. Como ressalta Large (2003, 2003, p. 122, tradução nossa): “As sensações não tem papel em uma teoria ecológica da percepção. $O$ enfoque ecológico da percepção baseia-se na informação." 25

Para exemplificar este trecho do livro de Large, podemos citar o caso da percepção do fogo. $\mathrm{O}$ fogo parece perigoso, porque percebemos diretamente a informação de que o calor e as chamas podem nos queimar. Para Large (2003), podemos aprender sobre o perigo que a chama nos proporciona, como queimar nossa pele, mas esse aprendizado não necessariamente é um processo de associação e representação do input sensorial do fogo e do output da queimadura. $\mathrm{O}$ fogo parece perigoso, porque aprendemos a captar diretamente a informação que específica que o fogo pode nos queimar.

Para Large (2003, p. 68-69), a percepção direta da informação é perpassada pelo efeito de ressonância. Sendo assim, a percepção não é um processo causal e linear, o qual expressa a unidirecionalidade da causa, mas um processo circular e recíproco que opera através do efeito de ressonância da informação ecológica. A ressonância ${ }^{26}$ não é caracterizada como um tipo de transmissão de impulsos dos órgãos dos sentidos para o cérebro, mas como informação que reverbera na percepção das invariantes e affordances que constituem a relação de reciprocidade entre organismo e ambiente. Nesse sentido, ela é caracterizada como ressonância ecológica, na medida em que é sistêmica, dinâmica e circular. Como ressalta Large (2003, p. 72-73, tradução nossa): "Sem essa ressonância não pode haver percepção. A ressonância proporciona o "como" da percepção. É como opera a percepção ecológica. [...] Ressonância perceptiva é uma

\footnotetext{
25 "Sensations has no role in an ecological theory of perception. The ecological approach to perception is information based." (LARGE, p. 122).

$26 \mathrm{O}$ conceito de ressonância é complexo. Neste trabalho não nos propomos a abordar toda a complexidade desse conceito, mas apenas expressar brevemente a sua relação com o reconhecimento de lugar, no viés ecológico gibsoniano.
}

MORONI, Juliana. Self e conteúdo não conceitual da percepção: a perspectiva anti-representacionista acerca da experiência perceptiva do reconhecimento de lugar. Griot : Revista de Filosofia, Amargosa, Bahia, v.16, n.2, p.285-302, dezembro/2017. 297 
propriedade da relação de reciprocidade entre o perceptor e o ambiente do perceptor." ${ }^{27}$ É através do efeito de ressonância ${ }^{28}$ que o organismo apreende diretamente a informação do ambiente que indica as regularidades e os padrões invariantes de similaridade e familiaridade, o que constitui a memória ecológica. Através da percepção das invariantes e da ressonância, forma-se a memória ecológica, a qual é responsável pelo reconhecimento do lugar. A memória ecológica emerge através da relação entre as propriedades do corpo e as propriedades do ambiente, via prontidão para a ação que é baseada na ressonância e reciprocidade.

Desse modo, questionamos: como podemos nos lembrar de alguém que conhecemos em determinado lugar, se ao lembrarmos dessa pessoa, estamos em outro lugar? Em outras palavras, como posso me lembrar de alguém que conheci no Brasil se eu estiver na Holanda? Entendemos que através da memória ecológica, caracterizada anteriormente no caso do reconhecimento de lugar, também podemos nos lembrar de alguém que não esteja presente sem recorrer à percepção via representações mentais. Isso porque a interação entre as propriedades informacionais (invariantes) do ambiente e as do organismo incorporado e situado possibilita a percepção de affordances presentes no âmbito da percepção-ação. No caso da lembrança de alguém que não está presente, imaginemos uma sala com algumas pessoas. As propriedades informacionais do corpo de determinada pessoa na sala, como a estrutura do seu corpo (invariantes estruturais) e o modo como essa pessoa se locomove no ambiente, podem nos remeter, via percepção direta, às invariantes semelhantes da pessoa com a qual temos algum tipo de relação e que não está presente. Desse modo, tais invariantes possibilitam a percepção de possibilidades de ação (affordances) no ambiente relacionadas às pessoas que estão na sala e à pessoa que não está presente, graças à interação entre as propriedades informacionais dos indivíduos presentes e os da pessoa ausente. Nesse caso, a memória ecológica, num primeiro momento, possibilita ao corpo o reconhecimento de lugar e a lembrança de pessoas ausentes, sem a necessidade de representações mentais.

Consideramos também que, assim como no caso do reconhecimento de lugar, a memória, de modo geral, é explicada através da percepção de padrões invariantes disponíveis no ambiente. Tais padrões são associados à concepção de padrão que liga, uma das bases conceituais da epistemologia ecológica proposta por Bateson (1986). Para ele, o padrão que liga é um metapadrão, sendo caracterizado como padrão dos padrões. (BATESON, 1986, p.19). Tais padrões são definidos como conexões de primeira, segunda e terceira ordem. Para Bateson (1986) esses metapadrões ressaltam as similaridades entre as partes de um mesmo indivíduo (conexão de primeira ordem); as partes de organismos diferentes (conexão de segunda ordem) e a relação dos organismos com o meio (conexão de terceira ordem). Essas similaridades não são definidas em termos de quantidade, mas caracterizadas em termos de relações, contornos e formas.

Assim, por exemplo, encontramos similaridades formais entre os ossos dos braços e das pernas de um ser humano (conexão de primeira ordem) e entre os ossos de um ser humano e de um cavalo (conexão de segunda ordem). (BATESON, 1986, p. 17-19). Os padrões que constituem os organismos e seus nichos contêm informações que, se inseridas em um contexto, direcionam a ação dos organismos e revelam suas histórias evolutivas. Essas histórias são

\footnotetext{
27 Without this resonance there can be no perception. Resonance provides the "how" of perception. It is how the ecological perception operates. [...] Perceptual resonance is a property of the reciprocal relationship between a perceiver and the perceiver's environment. (LARGE, 2003, p. 72-73).

${ }^{28}$ Um equívoco na concepção de ressonância é associá-la ao transdutor. Isso porque na concepção ecológica gibsoniana a ressonância é caracterizada como uma propriedade da relação de reciprocidade entre organismo e ambiente. Já na visão mecanicista da física clássica, o transdutor é um aparelho que transfere energia de um sistema para outro de formas iguais ou diferentes. (LARGE, 2003, p. 73). Para transferir e transformar a energia é utilizado um sensor. Nesse sistema mecânico não há qualquer relação de reciprocidade. Por exemplo, um sensor que transforma informação não elétrica (velocidade) em informação elétrica (corrente elétrica). (Fonte: http://pt.wikipedia.org/wiki/Transdutor).
}

MORONI, Juliana. Self e conteúdo não conceitual da percepção: a perspectiva anti-representacionista acerca da experiência perceptiva do reconhecimento de lugar. Griot : Revista de Filosofia, Amargosa, Bahia, v.16, n.2, p.285-302, dezembro/2017. 298 
construídas através da reciprocidade dinâmica entre agente e ambiente ecológico no qual o organismo deixa uma "marca" no meio e o meio, por sua vez, deixa uma "marca" no organismo. Essas marcas carregam a informação que especifica o organismo, sendo detectadas através do background do organismo em questão, isto é, de sua experiência vivida. Desse modo, ao perceber as invariantes e os padrões que ligam, percebemos as affordances, as quais expressam a informação de lugares, organismos e eventos.

\section{Considerações finais}

Iniciamos este trabalho com o objetivo de mostrar a relação entre Self, conteúdo não conceitual, percepção direta e reconhecimento de lugar, no viés ecológico gibsoniano e criticamente no viés ecológico proposto por Bermúdez. Primeiramente, expomos a perspectiva ecológica, proposta por Bermúdez, para posteriormente comparar as concepções de Bermúdez e de Gibson no que concerne ao reconhecimento de lugar.

Concordamos com Bermúdez (1994) de que não é necessário o conceito de primeira pessoa para o reconhecimento do lugar. Porém, problematizamos a posição de Bermúdez (1994) que considera necessário a consciência para o reconhecimento de lugar. Nossa proposta é a de que o reconhecimento de lugar pode ser feito através de estados primitivos e avançados de consciência, entretanto, tais estados não são necessários para o reconhecimento de lugar. Este pode ser realizado através da prontidão para a ação.

Nesse sentido, argumentamos que o reconhecimento de lugar ocorre através da memória ecológica, a qual caracterizamos como emergente da relação entre propriedades informacionais do ambiente e propriedades informacionais dos organismos. Dessa relação surgem padrões que ligam, caracterizados como as bases conceituais da epistemologia ecológica proposta por Bateson (1986). Esses padrões que ligam indicam as histórias co-evolutivas entre organismo e ambiente e carregam as informações que especificam a experiência vivida e compartilhada pelos organismos nos seus nichos.

Concluímos este trabalho com a proposta de que o reconhecimento do lugar une, através da percepção direta, passado e presente de forma contínua, sem que isso nos remeta a um estágio elevado de percepção que envolva a consciência, mesmo que essa seja concebida nos moldes primitivos, como defende Bermúdez. Além dessa união contínua, a qual denominamos memória ecológica, o reconhecimento do lugar envolve o estado de prontidão para a ação, a cinestesia, a ressonância e os padrões que ligam. Discordamos de Bermúdez de que há necessariamente um modo não conceitual primitivo de representação do Self que envolve consciência. Nossa hipótese é que este modo primitivo de percepção não envolve necessariamente a consciência para o reconhecimento do lugar. ${ }^{29}$ Prosseguiremos com nossas pesquisas sobre a relação entre Self não

\footnotetext{
29 Pesquisas sobre memória expressam a possibilidade dos seres humanos possuírem memória genética no âmbito cognitivo/semântico e de procedimento/hábito. Mas o que seria, mais precisamente, a memória genética? Para Darold Treffert, ela é caracterizada como habilidades herdadas que expressam complexidade e conhecimento sofisticado. A memória genética não é adquirida pela experiência, mas é congênita. Alguns pesquisadores contribuíram e contribuem para os estudos em memória genética, tais como Jung e sua concepção de inconsciente coletivo; Michael Gazzaniga e sua concepção de que o cérebro está sob controle genético, possuindo dispositivos que se atualizam em contextos apropriados. Nesse sentido, estes dispositivos são estruturados de forma inata. Eles se realizam antes de percebermos uma ação. Steven Pinker e Brian Butterworth também endossam o conceito de memória genética, afirmando que o desenvolvimento humano não começa do zero (tábula rasa), mas através da transmissão de conhecimento sofisticado ligado à genética. De acordo com Treffert, exemplos de memória genética podem ser observados em humanos e em outros animais, como borboletas. No caso dos humanos, após acidentes ou após doenças que lesionam o cérebro, as pessoas demonstram habilidades que antes não possuíam em áreas como música, arte, matemática, etc. Ou ainda, grupos de pessoas pertencentes aos denominados Savantes, os quais mostram habilidades em relação a coisas que nunca experienciaram ou aprenderam, como tocar um instrumento musical, sem sequer ter tido uma única aula. Outro exemplo de conhecimento genético, inato e complexo está relacionado às borboletas e suas habilidades de completar o caminho de ida e volta durante sua migração. O caminho de ida e de volta do Canadá até o México leva 3 gerações de borboletas. Desse modo,
}

MORONI, Juliana. Self e conteúdo não conceitual da percepção: a perspectiva anti-representacionista acerca da experiência perceptiva do reconhecimento de lugar. Griot : Revista de Filosofia, Amargosa, Bahia, v.16, n.2, p.285-302, dezembro/2017. 299 
conceitual e prontidão para ação a fim de obtermos subsídios teóricos para a elaboração de trabalhos posteriores que visam ampliar os nossos estudos.

nenhuma borboleta que vai, volta. A pergunta que é suscitada diante deste fato é: como elas sabem o caminho de volta? Para Treffert, essa habilidade complexa é atribuída à memória genética e ao conhecimento herdado que ela proporciona. Para Treffert, a memória genética existe nos organismos e o desafio que se impõe, por exemplo, no caso dos humanos, é saber como ativar a capacidade que está inativa sem causar lesões no cérebro. Entretanto, argumentos contrários expressam que alguns organismos têm tendência a desenvolverem e a absorverem o aprendizado mais rapidamente do que outros nas mesmas situações. $\mathrm{O}$ conhecimento, neste caso, não seria inato, mas vindo de estímulos do ambiente. Já para Treffert, o conhecimento é inato e precede o aprendizado. Não é nosso propósito, neste trabalho, abordar detalhadamente o conceito de memória genética, mas apenas indicar outras pesquisas sobre memória. (Fonte: http://blogs.scientificamerican.com/guest-blog/genetic-memoryhow-we-know-things-we-never-learnedl/).

MORONI, Juliana. Self e conteúdo não conceitual da percepção: a perspectiva anti-representacionista acerca da experiência perceptiva do reconhecimento de lugar. Griot : Revista de Filosofia, Amargosa, Bahia, v.16, n.2, p.285-302, dezembro/2017. 300 


\section{Referências bibliográficas:}

BATESON, Gregory. Mente e natureza. Rio de Janeiro: F. Alves, 1986.

BERMÚDEZ, José luis. Nonconceptual self-counciousness and cognitive science. In: Synthese, V. 129, n. 1, p. 129-149, 2001.

BERMÚDEZ, José luis; MARCEL, Anthony. ; EILAN, Naomi. The body and the self. Massachussetts: MIT, 1998.

BROENS, Mariana Claudia. Sujeito, teoria da auto-organização e o problema da identidade pessoal. In: Sujeito e Identidade Pessoal: Estudos de Filosofia da Mente. BOENS, M. C.; MILIDONI, C. B. (ORGS). São Paulo: Cultura Acadêmica, 2003, p. 113-126.

BROENS, Mariana Claudia. Algumas considerações sobre o conceito cartesiano de memória: um convite ao estudo interdisciplinar nas ciências cognitivas. In: Encontro com as ciências cognitivas. Cultura acadêmica: São Paulo, 2001, p. 27-34.

BROENS, Mariana Claudia.; GONZALEZ, Maria Eunice Quilici; MILLIDONI, Beatriz; QUILICIGONZALEZ, José Arthur. Filosofia da Mente e o pós-humano: para onde vamos com os novos desdobramentos do mecanicismo? No prelo. 2015.

BUTERWORTH, George. The noncenptual self. In: Human Development, p. 217-219, 1999.

CARELLO, Claudia; MICHAELS, Claire. Direct perception. USA: Prentice-Hall, Englewood cliffs, 1981.

CROWTHER, Thomas. Two conceptions of conceptualism and nonconceptualism. In: Erkenntnis, p. 245-276, 2006.

DEBRUN, Michel. A Idéia de Auto-Organização. In: Identidade nacional brasileira e auto-organização. (Orgs.) D'OTTAVIANO, I. L.; GONZALEZ, M.E.Q. Campinas: Coleção CLE. 2009. p. 53-74.

DEROY, Ophelia.; AUVRAY, Malika. How do synesthetes experience the world? In: M. Matthen (Ed.) Oxford Handbook of Philosophy of Perception, Oxford University Press (forthcoming).

DEROY, Ophelia.; AUVRAY, Malika. Beyond vision: The vertical integration of sensory substitution devices. In: M.Matthen \& D.Stokes (Ed,), Perception and its modalities, Oxford University Press, (2015).

GALLAGHER, Shaun. Philosophical conceptions of the self: implications for cognitive science. In: Trends in cognitive science, p. 13-21, 2000.

GALLESE, Vitorio.; SINIGAGLIA, Corrado. How the body in action shapes the self. In:Journal of Consciousness Studies, 2011, p.117-143.

GIBSON, James Jerome. The Ecological Approach to visual perception. New Jersey: Lawrence Earlbaum Associates, Inc, 1986.

GIBSON, James. Jerome. Reasons for realism. Lawrence Earlbaum Associates, Inc, 1982.

GIBSON, Eleonor. Ontogenesis of the perceived self. In: The perceived self: ecological and interpersonal sources of self-knowledge. Cambridge: Cambridge University Press, 1993, p. 25-42.

GONZALEZ, Maria Eunice Quilici.; HASELAGER, Willem (Pim). A identidade pessoal e a teoria da cognição incorporada e situada. BROENS, M. C.; MILIDONI, C. B. (Orgs). Sujeito e Identidade Pessoal: Estudos de Filosofia da Mente. São Paulo: Cultura Acadêmica, 2003, p. 95-111.

GONZALEZ, Maria Eunice Quilici; ANDRADE, Ramon. Informação e auto-organização: uma reflexão filosófica sobre a noção de significado. In: CARLOS CIRNE-LIMA; INÁCIO HELFER; LUIZ ROHDEN. (Org.). Dialética e Natureza. Caxias do Sul: Educs, p. 143-158,

2008.

GONZALEZ, Maria Eunice Quilici; MORAIS, Sonia.A teoria da percepção/ação e o comportamento sócio-cultural. GONZALEZ, M.E.Q.; FERREIRA, A.; COELHO, J. (Orgs.). Encontro com as Ciências Cognitivas V. São Paulo: Cultura Acadêmica, 2007, p. 149-161.

GONZALEZ, Maria Eunice Quilici.; BROENS, Mariana. Um estudo do conhecimento não proposicional no contexto da teoria da cognição incorporada e situada. In: Manuscrito - Rev. Int. Fil., , p. 729-751, 2006.

HASELAGER, Willem (Pim) \& GONZALEZ, Maria Eunice Quilici. A identidade pessoal e a Teoria da Cognição Situada e Incorporada. In: M.C. Broens, C.B. Milidoni, (Eds.). Sujeito e identidade pessoal: Estudos de Filosofia da Mente. São Paulo: Cultura Acadêmica, 2003, p. 95-11.

MORONI, Juliana. Self e conteúdo não conceitual da percepção: a perspectiva anti-representacionista acerca da experiência perceptiva do reconhecimento de lugar. Griot : Revista de Filosofia, Amargosa, Bahia, v.16, n.2, p.285-302, dezembro/2017. 301 
HEFT, Harry. The social constitution of perceiver environment reciprocity. Ecological Psychology. V. 19 , n. 2, p. 85-105, 2007.

LARGE, David. What is ecological philosophy? Disponível em: http://www.newphilsoc.org.uk/OldWebl/Ecological/what_is_ecological_philosophy.htm. Acesso em: 17 de maio de 2010.

LARGE, David. Ecological philosophy. Web Version. 2003. Disponível em: http://www.newphilsoc.org.uk/Ecological/DavidLarge.PDF. Acesso em: 10 de dezembrro de 2007. Traduzido por: MORONI, Juliana.; GONZALEZ, Maria Eunice Quilici; MORAES, João Antônio. O que é filosofia ecológica. In: Kínesis, Vol. III, n 05, Julho, p. 349-355, 2011.

LOW, Philip.; PANKSEPP, Jaak.; REISS, Diana.; EDELMAN, David.; VAN SWINDEREN, Bruno. The Cambridge Declaration on Consciousness. 2012. Disponível em: http://www.allcreatures.org/articles/ar-conscious.pdf. Acesso em: 07 de outubro de 2015.

MORONI, Juliana. Epistemologia Ecológica: a concepção de uma nova teoria do conhecimento proposta por Gregory Bateson. Filogênese. Marília: UNESP, p. 259-267, 2008. Disponível em: http:/www.marilia.unesp.br/Home/RevistasEletronicas/FILOGENESE//Juliana\%20Moroni\%20\%2030\%20_259-267_.pdf. Acesso em: 7 de maio de 2011.

MORONI, Juliana. Uma reflexão filosófica sobre o conceito de informação ecológica. Dissertação de mestrado apresentada ao Programa de Pós- Graduação em Filosofia da Universidade Estadual Paulista, Campus de Marília, como parte dos requisitos para a obtenção do título de Mestre em Filosofia, 2012.

MORONI, Juliana. Estudo epistemológico do conceito de informação no âmbito das vertentes cibernética, ecológica e semântica. Monografia apresentada ao Departamento de Filosofia da Faculdade de Filosofia e Ciências da Universidade Estadual Paulista, Campus de Marília como parte dos requisitos para a obtenção do título de Bacharel em Filosofia. 2008.

NEISSER, Ulric. The self perceived. In: The perceived self: ecological and interpersonal sources of selfknowledge. Cambridge: Cambridge University Press. 1993.

NEISSER, Ulric. Criteria for an ecological self. In: Advances in Psychology: The Self in Infancy- Theory and Research. V. 112, P. 17-34, 1995.

NEISSER, Ulric. Five kinds of self-knowledge. In:Philosophical Psychology. Taylor \& Francis. V. 1,p.35$59,1988$.

PEACOCKE, Christopher. Does Perception Have a Nonconceptual Content? In: The Journal of Philosophy, 239-264, 2001.

TREFFERT, Darold. Genetic-memory-how-we-know-things-we-never-learned. In: Scientific American, Jan. 2015. Acesso em 22/05/2015. Disponível em: http://blogs.scientificamerican.com/guest-blog/geneticmemory-how-we-know-things-we-never-learnedl/

VAZ, Daniela. Direct perception requires an animal-dependent concept of specificity and of information. In: Ecological Psychology. Routledge. 2015, v. 27, p. 144-174.

Autor(a) para correspondência: Juliana Moroni, Universidade do Estado do Rio de Janeiro, R. São Francisco Xavier, 524, Maracanã, CEP 20550-900, Rio de Janeiro - RJ, Brasil. julianamoroni@yahoo.com.br 